\title{
Probing confined phonon modes in individual CdSe nanoplatelets using surface-enhanced Raman scattering
}

\author{
Daniel O. Sigle, ${ }^{1}$ James T. Hugall, ${ }^{1}$ Sandrine Ithurria, ${ }^{2}$ Benoit Dubertret, ${ }^{2}$ \\ and Jeremy J. Baumberg ${ }^{1, *}$ \\ ${ }^{1}$ NanoPhotonics Centre, Cavendish Laboratory, University of Cambridge, Cambridge, CB3 \\ OHE, United Kingdom \\ ${ }^{2}$ Laboratoire de Physique et d'Etude des Matériaux, UMR8213 du CNRS, 10 rue Vauquelin \\ 75231 Paris, France
}

(Dated: December 18, 2013)

\begin{abstract}
The phonon modes of individual ultrathin cadmium selenide (CdSe) nanoplatelets are investigated using surface-enhanced Raman scattering in a tightly-confined plasmonic geometry. The SERS spectra, taken on single nanoplatelets sandwiched between a gold nanoparticle and a gold surface, reveal a phonon doublet arising from oscillations perpendicular to, and within the platelet plane. The out-of-plane mode cannot be observed with conventional Raman spectroscopy. The resulting strong electric field enhancements and the field vector reorientation within such nanometresized plasmonic gaps reveal otherwise hidden information deep into the Brillouin zone illuminating the vibrational properties of ultrathin materials.
\end{abstract}

PACS numbers: 73.20.Mf, 63.22.-m, 78.30.-j, 81.07.St 
Metallic nanoparticles in close proximity support strong electromagnetic fields confined in the gap between them $[1,2]$. Such plasmonic nanogaps are of increasing interest due to their ability to strongly enhance optical processes such as Raman scattering, in order to detect trace concentrations of substances with sensitivities down to the single molecule level [3-6]. Colloidal nanoplatelets [7-9], consisting of a thin (multi-atomic-layer) crystalline slab of inorganic material, represent an atomic system with high spatial confinement in a single dimension. Nanoplatelets have been grown from a wide variety of materials, but those made from semiconductors, which act as two-dimensional quantum wells, give particularly interesting optical properties. Their photoluminescence can be precisely tuned by varying the number of atomic layers in the slab, thus altering the spatial confinement of excitons and their resulting confinement and binding energies. Planar semiconducting nanoplatelets are thus useful to a broad range of disciplines ranging from solar cells and single-photon sources to molecular tagging and sensing. As well as exciton confinement, the phonons inside these ultra-thin semiconductor structures also feel confinement effects.

Here we demonstrate that plasmonic field localisation can be used to study the phonon confinement in extremely thin individual nanoplatelets consisting of a handful of atomic layers. Besides electric field-enhancement, the reorientation of the electric field vector perpendicular to the surface allows access to highly confined phonon modes not visible in standard Raman spectroscopy. Despite extensive research on Raman scattering of nanocrystals [10-15], there has been little focus on the surface-enhanced Raman scattering (SERS) of colloidal quantum dots [16-18]. Until now no reports exist of SERS of individual semiconducting nanoparticles. We investigate the Raman scattering of individual CdSe nanoplatelets [7] sandwiched between a gold nanoparticle (AuNP) and a planar gold surface. The nanoparticle on mirror geometry (NPoM) [19-21] enables highly-effective plasmonic coupling and convenient investigation of a single plasmonic junction, allowing the interrogation of single nanoplatelets. The dramatic change in the electric-field distribution in such a nanometre-sized gap and the resulting modification of traditional Raman selection rules allow us to probe phonon generation in CdSe nanoplatelets with different spatial dimensions. In this SERS configuration, we observe a highly-confined longitudinal optical (LO) phonon mode perpendicular to the platelet plane which is invisible in conventional Raman scattering. The spectral position of this out-of-plane $[\mathrm{LO}(z)]$ phonon is in agreement with existing phonon confinement models for spherical nanocrystals [12-14,22]. This work 
highlights the use of SERS within plasmonic nanogaps to not only provide a dramatic field enhancement but also reveals modes which are not visible in conventional Raman scattering.

Samples for SERS were prepared as follows: Colloidal nanoplatelets were grown by wet chemistry [6]. A $70 \mathrm{~nm}$ thick gold film was electron-beam evaporated on a silicon wafer, and then functionalized with a monolayer of 4-aminothiophenol (ATP) by immersion in a $10 \mathrm{mM}$ ethanolic solution for $24 \mathrm{~h}$. These prepared surfaces, rinsed clean and dried, were then dipped in colloidal solutions of nanoplatelets in hexane for another $24 \mathrm{~h}$. This gives a homogenous monolayer of platelets lying flat with a surface coverage of $\sim 60 \%$ as verified by AFM. Colloidal AuNPs with $100 \mathrm{~nm}$ diameter were drop-cast onto the surface, where they physisorb, and after 5 mins, unbound particles were washed away. For conventional Raman scattering, colloidal platelets were dropped on a clean silicon wafer and dried. Platelets in this case form stacks [23] which are in random orientations with respect to the surface. Raman spectra were measured on a Renishaw inVia Raman microscope at excitation wavelengths of $532 \mathrm{~nm}$ and $633 \mathrm{~nm}$. The microscope was set up in backscattering configuration and a 100x objective with $\mathrm{NA}=0.85$ was used for excitation and collection of scattered light. The incident light was unpolarized. The SERS spectra were collected from a single nanoparticle in the sandwich formation described above. This was ensured by a sufficiently sparse coverage of AuNPs on the surface (supporting information).

We analyse the Raman and SERS spectra taken in the NPoM geometry [Fig. 1(a)] for different CdSe platelets with 3, 4, and 5 atomic layers, which correspond to thicknesses of $1.064 \mathrm{~nm}, 1.368 \mathrm{~nm}$ and $1.672 \mathrm{~nm}$ [24]. The platelets investigated have a zinc blende lattice [6], rather different from the normal wurtzite structure for spherical nanoparticles. Besides their thickness, they also differ in their lateral dimensions. The 3-layer thick platelets are $\sim 200 \mathrm{~nm}$ long and $\sim 50 \mathrm{~nm}$ wide. The 4-monolayer platelets are $\sim 10 \mathrm{~nm} \times 10 \mathrm{~nm}$ and the 5layer platelets are $\sim 25 \mathrm{~nm} \times 7 \mathrm{~nm}$ [Fig. 1(b-d)]. Their bandgap wavelength is however defined only by their thickness [7] and corresponds to $460 \mathrm{~nm}$ for the 3 layer, $510 \mathrm{~nm}$ for the 4 layer and $550 \mathrm{~nm}$ for the 5 layer structures. 

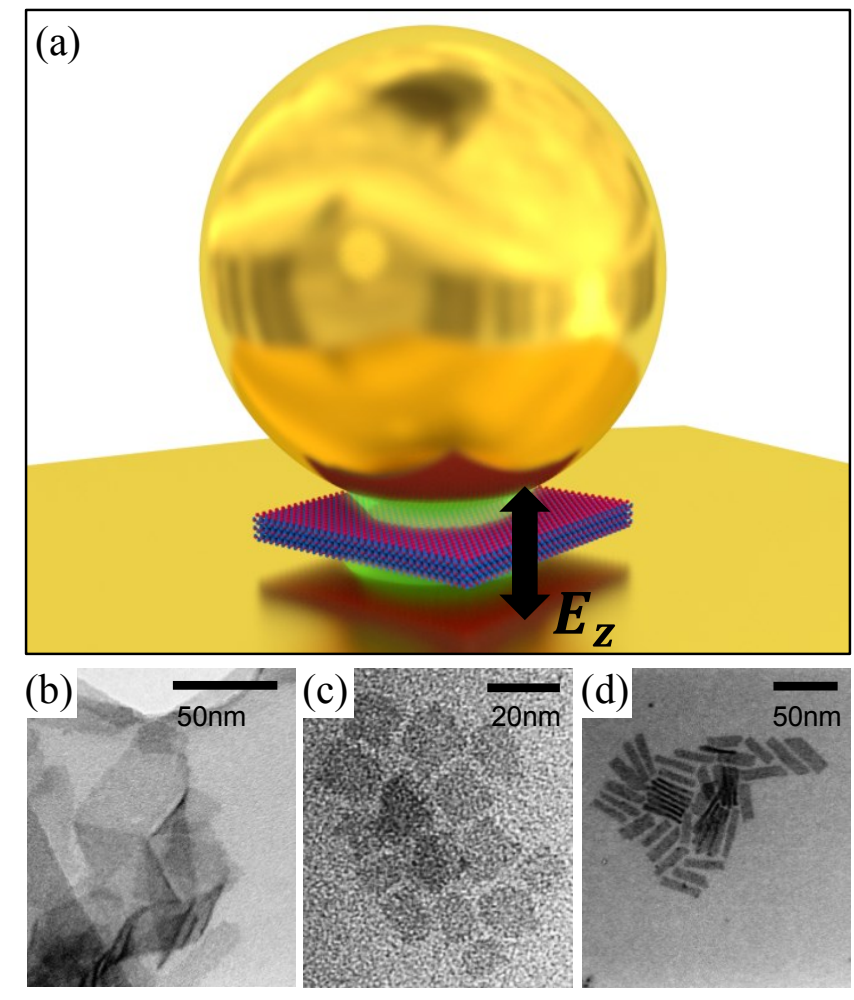

FIG. 1 (color online). (a) Individual CdSe platelet in a nanogap. (b-d) TEM of CdSe platelets with (b) 3 , (c) 4, and (d) 5 atomic layers.

The SERS spectra typically show the LO mode near $200 \mathrm{~cm}^{-1}$ [25] and a weak signature of the first overtone 2(LO). In addition to these modes, the bulk Raman spectra reveals the 3(LO) as well as the surface mode (SO) at $\sim 180 \mathrm{~cm}^{-1}$ appearing as a weak shoulder (not shown). The surface mode is strongest for the 3 layer platelets and diminishes for increasing platelet thickness as a result of decreasing surface to volume ratio. A minor peak is seen near $270 \mathrm{~cm}^{-1}$, which is identified as cadmium sulphide [26] and most likely stems from interaction with the thiols in the ATP. We focus discussions on the LO mode and start with observations made on the 4-monolayer thick platelets with near-resonant excitation at 532 $\mathrm{nm}$. The SERS in the NPoM geometry shows a strong peak of the LO phonon mode at 200 $\mathrm{cm}^{-1}$, while in the bulk Raman spectrum taken on the silicon wafer this mode appears at 206 $\mathrm{cm}^{-1}$ [Fig. 2(a,c)]. The $200 \mathrm{~cm}^{-1}$ SERS peak is attributed to a phonon with wavevector perpendicular to the platelet plane $[\mathrm{LO}(z)]$ whose strong spatial confinement leads to a spectral shift with respect to the bulk CdSe LO phonon $\left(\sim 210 \mathrm{~cm}^{-1}\right)$. The Raman peak at 206 $\mathrm{cm}^{-1}$ mainly originates from a phonon with wavevector directed along the platelet plane $[\mathrm{LO}(x)]$ which is far less spatially confined. The selective excitation of the out-of-plane 
phonon in the SERS is explained by the strong vertical electric field polarization in the nanogap within the NPoM assembly, hence perpendicular to the nanoplatelet [18]. The strongly confined optical field enables coupling to the out-of-plane phonon mode. In conventional Raman scattering, the spectrum is dominated by the in-plane mode, which is present over a far larger surface area and hence has a larger interaction cross-section with the incident light. This is also confirmed by the Raman selection rules. With the $z$-direction normal to the (001) platelet face [27], the $\operatorname{LO}(x)$ mode is allowed in the backscattering geometry $z\left(x^{\prime}, x^{\prime}\right) \bar{z}$, whereas $\mathrm{LO}(z)$ is forbidden (as observed away from the NPoM). Inside the nanogap the selection rules are changed because effectively the excitation geometry becomes $x\left(z^{\prime}, z^{\prime}\right) \bar{x}$ and $\mathrm{LO}(z)$ becomes allowed.

(a)

(c)

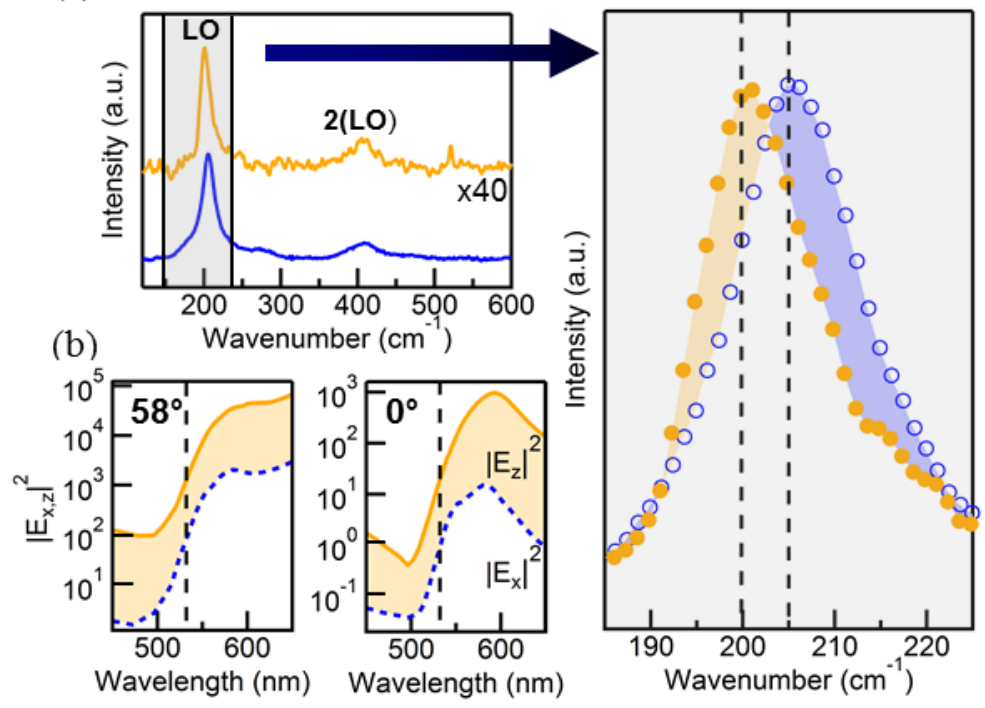

FIG. 2 (color online). (a) SERS spectrum from single NPoM geometry (top, yellow) and corresponding bulk Raman spectrum (bottom, blue) of 4 layer thick CdSe platelets (SERS trace is offset and multiplied by 40 for clarity). (b) Field components $\left|E_{z}\right|^{2}$ (yellow, solid line) and $\left|E_{x}\right|^{2}$ (blue, dashed line) in the nanogap. Left graph is for $58^{\circ}$ incidence (max. illumination angle for $\mathrm{NA}=0.85$ ), right for normal incidence. Black dashed line indicates Raman excitation. (c) Magnified spectra of LO phonon mode for bulk Raman (blue, empty circles) and SERS (yellow, solid circles) with intensities normalized.

To calculate the SERS enhancement factors we collect Raman scattered light from a diffraction limited spot on a monolayer of CdSe platelets on the planar gold surface and compare to that from a location containing a single gold NP on top of one of the CdSe 
platelets. The collected Raman scattered light for $532 \mathrm{~nm}$ excitation is approximately five times higher for the case with the AuNP, due to the creation of a localised electric field hotspot within a single platelet. Taking into account that only the plasmonic junction (whose lateral field diameter is given by $w=\sqrt{R d}$ for NP radius $R$ and gap size $d$ [28]) contributes to this enhancement while the collection area is $1 \mu \mathrm{m}$ in diameter, we obtain an actual SERS enhancement inside the gap of $\sim 5 \times 10^{4}$. The field in the nanogap is strongly polarized along the $z$-axis within the range of excitation angles given by the numerical aperture, as qualitatively found by modeling the system with different angles of incidence using the boundary-element method. In Fig. 2(b), the simulated near-field components in the platelet plane $(x)$ and vertical to the platelet plane $(z)$ are shown for normal incidence $\left(\left|E_{z}\right|^{2}=10\right.$, $\left|E_{x}\right|^{2}=1$ at the Raman excitation line) and the maximum angle of $58^{\circ}\left(\left|E_{z}\right|^{2}=10^{3},\left|E_{x}\right|^{2}=10^{2}\right)$. This ten-fold larger $|E|^{2}$ results in 2 orders of magnitude higher field enhancement in the zdirection (as SERS scales as $|E|^{4}$ ). Despite neglecting irregularities of the NP shape as well as possible chemical enhancement effects, the experimental findings are well supported.

(a)

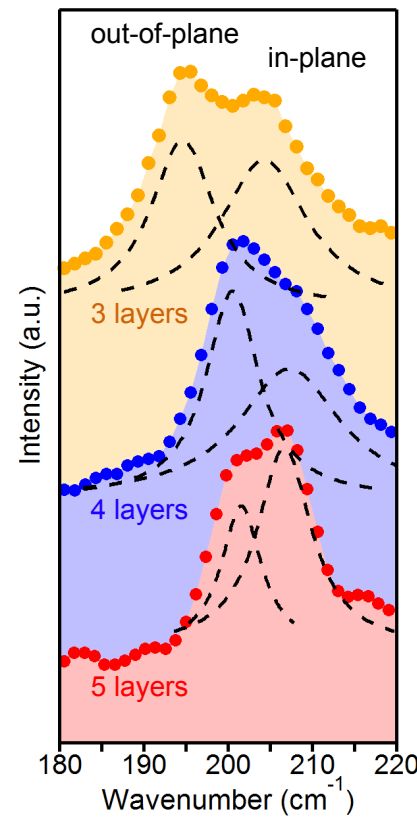

(b)

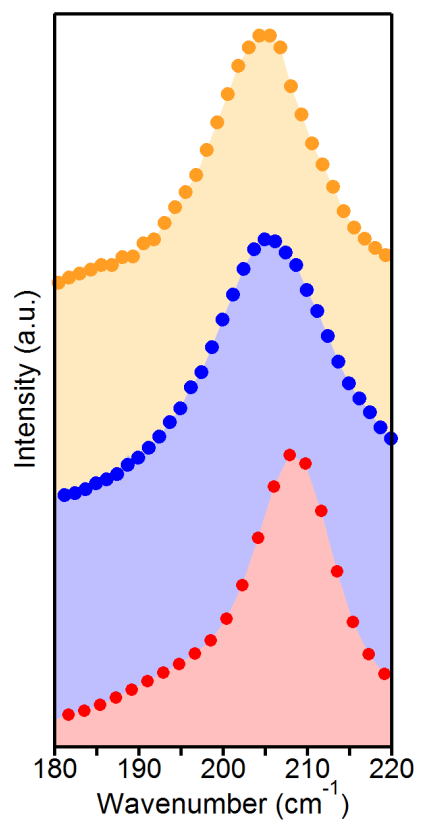

FIG. 3 (color online). (a) SERS of CdSe nanoplatelets with different thickness: 3, 4 and 5 atomic layers. (b) Conventional Raman scattering of a bulk film of the same platelets. Spectra are averaged over $\sim 10$ spectra from different nanoparticles and offset for clarity. 
To reveal the confinement-dependent properties of the phonon modes we compared nanoplatelets of three different thicknesses with 3, 4 and 5 atomic layers. While the 3 layer crystals were pumped with $532 \mathrm{~nm}$ analogous to the 4 layer sample, we had to employ an excitation wavelength of $633 \mathrm{~nm}$ for the 5 layer platelets, to avoid the strong photoluminescence which masks the Raman peaks. While SERS of the 4 layer platelets shows the out-of-plane phonon more prominently than the in-plane mode, both modes have comparable intensities for the 3 and 5 monolayer systems [Fig. 3(a)]. By contrast, the bulk Raman spectra contain only one peak in all three cases, identified as the in-plane mode [Fig. 3(b)]. The relatively smaller excitation of out-of-plane amplitudes on the 3 and 5 monolayers is likely because the 4-layer system is excited closer to the excitonic resonance, resulting in resonant Raman scattering (SERRS) with more efficient coupling to the LO phonon modes. While bulk Raman scattering on arrays of such CdSe platelets showed recently [11] that spectral tuning with respect to the exciton determines which phonon modes are observed, here the geometry of the extreme plasmonic enhancement on single nanoparticle constructs is instead responsible. While the spectral mode positions remain highly reproducible, the intensity ratio between in- and out-of-plane modes fluctuates between different nanoparticle sandwiches. Nanoscale deviations of each AuNP from a spherical shape lead to a dramatic variation in the plasmonic resonance conditions for such extreme confinement, and thus the observed enhancement.

(a)

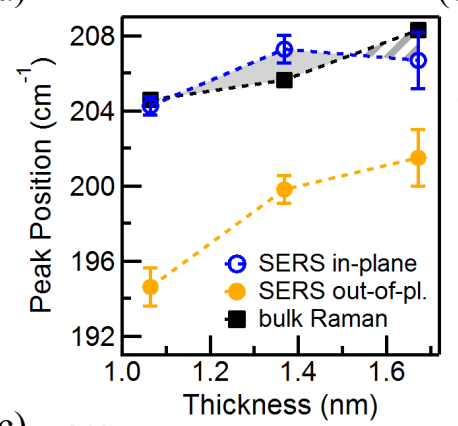

(b)

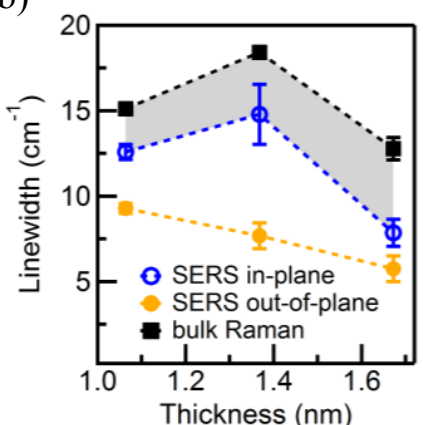

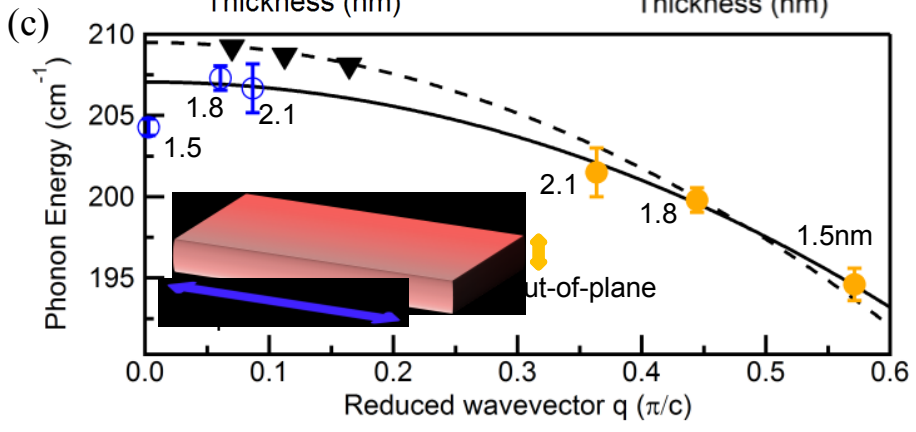


FIG. 4 (color online). (a) Peak positions and (b) linewidths (FWHM) of SERS and bulk Raman. (c) Phonon energies vs. reduced wavevector (in units of the lattice constant). Yellow points are the outof-plane mode with black curve fit to Eq. (1). Blue circles show the in-plane mode. Black triangles and dashed black curve are data points and fit for spherical nanocrystals from Hwang et al. [13].

The spectral mode positions for the SERS and bulk Raman experiments are shown in Fig. 4(a). The out-of-plane $\operatorname{LO}(z)$ mode redshifts strongly by $7 \mathrm{~cm}^{-1}$ between the 5 and 3 layer platelets while the in-plane modes are much less sensitive to the platelet geometry. The energies of the in-plane mode in SERS and bulk Raman do not exactly match for the 4 and 5 layer platelets, most likely due to additional out-of-plane contributions to the bulk Raman spectra which induce broadening and slight line asymmetries [Fig. 4(b)].

The phonon shift is explained by two contributions. First, the negative dispersion of the LO-branch away from the zone-centre causes a redshift with increasing phonon confinement (which accesses higher phonon wavevectors). The second contribution arises from the contraction of the crystal lattice near the surface $[12,13]$. In order to determine the contribution of the negative dispersion, we consider the platelets as a 1-dimensional quantum well. The phonon eigenfrequencies can be expressed by [14]

$$
\omega_{n, \mathrm{LO}}^{2}=\omega_{\mathrm{bulk}, \mathrm{LO}}^{2}-\beta_{\mathrm{LO}}^{2} q_{n}^{2}
$$

The crystal momentum of the integer $n^{\text {th }}$ resonance under spatial confinement with a platelet thickness $d$ is given by

$$
q_{n}=\frac{n \pi}{d}
$$

The parameter $\beta_{\mathrm{LO}}$ describes the dispersion relation of the LO branch which is assumed to be quadratic in the long wavelength limit where the relevant lattice parameter $c=0.61 \mathrm{~nm}$.

Fitting the strongly confined out-of-plane modes [Fig. 4(c), yellow points] and the inplane modes of 4- and 5-layer platelets (blue circles) yields $\omega_{\text {bulk,LO }}=207.1 \mathrm{~cm}^{-1}$ and $\beta_{\mathrm{LO}}=2.27 \times 10^{3} \mathrm{~m} / \mathrm{s}$. We note that the in-plane mode of the 3 layer platelets is redshifted compared to the predictions of this confinement model. As the 3 layer platelets are much longer $(\sim 200 \mathrm{~nm})$ than the other two types, geometric defects can occur such as curling and folding [Fig. 1(c)] [27] which can account for these redshifts. Previous data is only available 
on spherical quantum dots in the wurtzite lattice structure. Hwang and Park [13] suggest $\beta_{\mathrm{LO}}=2.97 \times 10^{3} \mathrm{~m} / \mathrm{s}$ although their data reaches much less far out from the zone centre (and hence producing five-fold smaller energy shifts, data added to Fig. 4(c) with fit dashed). For the spherical crystals, we use $q_{n}=2 \mu_{n} / d$ [14], with $\mu_{n}$ as the $n^{\text {th }}$ node of the $J_{1}$ Bessel function, and a lattice parameter $c=0.70 \mathrm{~nm}$. The discrepancy between our data and the spherical crystals originates in the different lattice geometry (zinc blende) as well as a higher lattice contraction in the platelets. In our case, the phonons travel in close proximity along the crystal surface where the strain reaches a maximum. We also note that the phonon dispersion in bulk wurtzite CdSe measured by inelastic neutron scattering [29] yields $\beta_{\mathrm{LO}}=2.48 \times$ $10^{3} \mathrm{~m} / \mathrm{s}$, which is comparable to the measurements above, but the appropriate zinc blende structure cannot be stabilised in the bulk.

The linewidths of out-of-plane and in-plane modes exhibit different thickness dependences. The higher spatial confinement of the out-of-plane mode for decreasing platelet thickness results in a stronger phonon-phonon interaction and subsequent relaxation at the boundaries of the platelets. This leads to a near doubling of the FWHM linewidth from 5.7 $\mathrm{cm}^{-1}$ to $9.2 \mathrm{~cm}^{-1}$ in the 3- to 5-layer platelets [Fig. 4(b)]. The linewidth of the in-plane phonons follows a different dependence but is largest on the 4 monolayer platelets, probably because of the different near-resonant excitation condition. These changes in linewidth for different phonon confinement track the increasingly-large phonon wavevectors involved [Fig. $4(\mathrm{c})]$.

In conclusion, we demonstrate the capability of studying SERS on single ultrathin semiconductor platelets of different numbers of monolayers. Using the extreme plasmonic confinement in the nanoparticle-on-mirror NPoM geometry accesses for the first time the SERS of individual semiconductor nanostructures. The measurements reveal an energy splitting between in-plane and out-of-plane LO phonons in these CdSe nanoplatelets, not observable in conventional Raman spectroscopy. The spectral position of the out-of-plane component is highly sensitive to the thickness of the platelet in agreement with a phononconfinement model. The concept of spatially confined phonons can thus be used for noninvasive thickness probing of nanometre-thin semiconductor films with Angstrom-level precision. This is of great interest to a broad range of nanofabrication technologies where accurate film thickness control is required. This also opens the potential to excite and track local phonon interactions within and in neighbouring nanostructures. Finally the extreme 
plasmonic confinement and ultrathin layers access phonon wavevectors deeply into the Brillouin zone, allowing new probes of phonon coupling and decay. A wide range of ultrathin semiconductors can be utilised, for instance $\mathrm{MoS}_{2}, \mathrm{WSe}_{2}$, as well as many others, which opens up exciting prospects for plasmon-phonon coupling at the nanoscale.

This work was supported by the UK EPSRC grant EP/G060649/1, Defence Science and Technology Laboratory (DSTL), and ERC grant 320503 LINASS.

*jjb12@cam.ac.uk

\section{References:}

[1] R. Esteban, R. W. Taylor, J. J. Baumberg, and J. Aizpurua, Langmuir 28, 8881 (2012).

[2] R. W. Taylor, T.-C. Lee, O. A. Scherman, R. Esteban, J. Aizpurua, F. M. Huang, J. J. Baumberg, and S. Mahajan, ACS Nano 5, 3878 (2011).

[3] P. G. Etchegoin, E. C. Le Ru, A. Fainstein, E. Vela, and R. C. Salvarezza, J. Am. Chem. Soc. 135, 2809 (2013).

[4] S. Nie and S. R. Emory, Science 275, 1102 (1997).

[5] K. Kneipp, Y. Wang, H. Kneipp, L. Perelman, I. Itzkan, R. Dasari, and M. Feld, Phys. Rev. Lett. 78, 1667 (1997).

[6] E. C. Le Ru, M. Meyer, and P. G. Etchegoin, J. Phys. Chem. B 110, 1944 (2006).

[7] S. Ithurria, M. D. Tessier, B. Mahler, R. P. S. M. Lobo, B. Dubertret, and A. L. Efros, Nat. Mater. 10, 936 (2011).

[8] S. Ithurria and B. Dubertret, J. Am. Chem. Soc. 130, 16504 (2008).

[9] C. Bouet, M. D. Tessier, S. Ithurria, B. Mahler, B. Nadal, and B. Dubertret, Chem. Mater. 25, 1262 (2013).

[10] H. Lange, M. Artemyev, U. Woggon, and C. Thomsen, Nanotechnology 20, 045705 (2009).

[11] S. A. Cherevkov, A. V. Fedorov, M. V. Artemyev, A. V. Prudnikau, and A. V. Baranov, Phys. Rev. B 88, 041303 (2013).

[12] Y. Hwang, S. Shin, H. Park, S. Park, U. Kim, H. Jeong, E. Shin, and D. Kim, Phys. Rev. B 54, 15120 (1996). 
[13] Y.-N. Hwang, S.-H. Park, and D. Kim, Phys. Rev. B 59, 7285 (1999).

[14] C. Trallero-Giner, A. Debernardi, M. Cardona, E. Menendez-Proupin, and A. I. Ekimov, Phys. Rev. B 57, 4664 (1998).

[15] V. Dzhagan, M. Valakh, N. Mel'nik, O. Rayevska, I. Lokteva, J. Kolny-Olesiak, and D. R. T. Zahn, Int. J. Spectrosc. 2012, 1 (2012).

[16] J. T. Hugall, J. J. Baumberg, and S. Mahajan, Appl. Phys. Lett. 95, 141111 (2009).

[17] A. G. Milekhin, L. L. Sveshnikova, T. A. Duda, N. V. Surovtsev, S. V. Adichtchev, and D. R. T. Zahn, JETP Lett. 88, 799 (2009).

[18] A. Rumyantseva, S. Kostcheev, P.-M. Adam, S. V Gaponenko, S. V Vaschenko, O. S. Kulakovich, A. A. Ramanenka, D. V Guzatov, D. Korbutyak, V. Dzhagan, A. Stroyuk, and V. Shvalagin, ACS Nano 7, 3420 (2013).

[19] J. Mertens, A. L. Eiden, D. O. Sigle, F. Huang, A. Lombardo, Z. Sun, R. S. Sundaram, A. Colli, C. Tserkezis, J. Aizpurua, S. Milana, A. C. Ferrari, and J. J. Baumberg, Nano Lett. 13, 5033 (2013).

[20] C. Ciracì, R. T. Hill, J. J. Mock, Y. Urzhumov, A. I. Fernández-Domínguez, S. A. Maier, J. B. Pendry, A. Chilkoti, and D. R. Smith, Science 337, 1072 (2012).

[21] L. Li, T. Hutter, A. S. Finnemore, F. M. Huang, J. J. Baumberg, S. R. Elliott, U. Steiner, and S. Mahajan, Nano Lett. 12, 4242 (2012).

[22] H. Richter, Z. P. Wang, and L. Ley, Solid State Commun. 21, 625 (1981).

[23] M. D. Tessier, L. Bilada, C. Bouet, S. Ithurria, B. Abecassis, and B. Dubertret, ACS Nano 7, 3332 (2013).

[24] B. Mahler, B. Nadal, C. Bouet, G. Patriarche, and B. Dubertret, J. Am. Chem. Soc. 134, 18591 (2012).

[25] A. M. Kelley, Q. Dai, Z. Jiang, J. A. Baker, and D. F. Kelley, Chem. Phys. 422, 272 (2013).

[26] L. Lu, X.-L. Xu, W.-T. Liang, and H.-F. Lu, J. Phys. Condens. Matter 19, 406221 (2007).

[27] C. Bouet, B. Mahler, B. Nadal, B. Abecassis, M. D. Tessier, S. Ithurria, X. Xu, and B. Dubertret, Chem. Mater. 25, 639 (2013).

[28] K. J. Savage, M. M. Hawkeye, R. Esteban, A. G. Borisov, J. Aizpurua, and J. J. Baumberg, Nature 491, 574 (2012).

[29] F. Widulle, S. Kramp, N. M. Pyka, A. Goebel, T. Ruf, A. Debernardi, R. Lauck, and M. Cardona, Phys. B 263-264, 448 (1999). 
